\title{
Preparation and Characterization of Metal Complex Hydrogels Crosslinked with Hyperbranched Polyester
}

\author{
Ahmed A. Haroun ${ }^{* 1}$, O. A. Hakeim², Olga Trhlíkován ${ }^{3}$ Miroslav Šlouf ${ }^{3}$, Miloš \\ Netopilík ${ }^{3}$ \\ ${ }^{1}$ Chemical Industries Research Division, ${ }^{2}$ Textile Division, National Research Centre \\ 12622 Dokki, Giza, Egypt and ${ }^{3}$ Institute of Macromolecular Chemistry, ASCR,v.v.i, \\ Heyrovskho Sq.2, Prague 6,16206 Czech Republic.
}

\begin{abstract}
7 HIS WORK deals with preparation and characterization of metal complex hydrogels based on crosslinked chitosan with hyperbranched polyester generation 2 . These materials were prepared using miniemulsion technique with different ratios. Besides, the metal complexes using $\mathrm{Co}, \mathrm{Ni}$ and $\mathrm{Fe}$ salts were investigated. All the prepared materials were characterized using Fourier transform infrared spectroscopy (FTIR), thermogravimetric analysis (TGA), particle size and zeta potential analysis using dynamic light scattering (DLS) technique. Moreover, XRF was also carried out. The results indicated that the successful network structures were obtained with particle size ranged between 118 and $148 \mathrm{~nm}$. In addition, high performance metal complexes were prepared with metal percentages about $6.55,7.01$ and 6.56 (wt\%), in the case of $\mathrm{Co}, \mathrm{Ni}$ and $\mathrm{Fe}$, respectively.Moreover, thermal analysis revealed that hydrogen bonds played an important role for hydrogel networks formation.
\end{abstract}

Keywords: Hyperbranched polyester,Chitosan, Miniemulsion, Metal complexes, Hydrogels.

\section{Introduction}

The grafted nanosurfaces using hyperbranched polymers have become an attractive research field because of their multifunctional properties [1-3]. Moreover, hyperbranched polyesters (HBPE) were previously used as crosslinking agents for polyurethane networks to explore novel polymeric materials in industry [4]. The high branching results in high solubility, low viscosity and could be used as initiator for ring opening polymerization of lactones. Chitosan is a biodegradable renewable polymer obtained by deacetylation of chitin. It has an excellent metals binding capacity by its physical or chemical modifications [5-12]. On the other hand, it has some disadvantages such as: acidic solubilization which prevents its use as insoluble sorbent in this medium. Consequently, it must be modified via crosslinking reaction. Some crosslinking agents could be used such as: epichlorohydrin (EPI), and glutaraldehyde $[13,14]$.

Nowadays, the water resources in the lowincome countries suffer from the industrial effluents, which becomes a serious problem. Metal ion contamination has increased substantially because of the use of metal ions in many industrial applications. The functional polymeric composites have gained great importance in environmental applications for determining of toxic metal ions and their removal from aqueous media because of their high selectivity, excellent loading capacity and good recovery $[15,16]$. This work deals with preparation and characterization of metal complex hydrogels based on crosslinked chitosan with hyperbranched polyester generation 2 (HBPE) in the presence or in absence of acetate salts of cobalt, nickel and iron, separately. In other words, the study aims to enhance the hydrophilicity and metal binding ability of chitosan through incorporation of HBPE with 16-OH groups along chains using easy emulsion technique.

\section{Experimental}

Materials

Hyperbranched polyester generation 2 obtained from Sigma-Aldrich. Chitosan of low molecular

*Corresponding author e-mail: haroun68_2000@yahoo.com

DOI: 10.21608/ejchem.2017.1310.1081

C2017 National Information and Documentation Centre (NIDOC) 
weight was obtained from Aldrich. All other chemicals and reagents were used as received.

\section{Methods}

Preparation of metal complex hydrogels using emulsion technique

Emulsion experiments [17] were carried out in tubes after homogenizing with high speed sonification. Chitosan $(10 \mathrm{mg})$ was dissolved in aqueous acidic medium. The solution was mixed with different ratios (1:1 and 2:1) of HBPE in an aqueous solutions containing definite amounts of acetate salts of different metal salts (Co, Ni and $\mathrm{Fe}$ ), separately and emulsified in presence of cetyltrimethylammonium bromide(CTAB) using an ultrasonifier ( $3 \mathrm{~min}$ at $200 \mathrm{~W}$ ). In other experiments, the same ingredients were prepared without metal salts. The resulting hydrogels were separated using centrifuge, then collected and kept for further investigation, as shown in Table 1.

\section{Characterization}

All the prepared materials were characterized using different analytical tools:

1) Perkin-Elmer Fourier transform infrared spectroscopy (FTIR) under certain condition such as: scan resolution: $4 \mathrm{~cm}^{-1}$, scan rate: 2 $\mathrm{mm} \mathrm{sec}{ }^{-1}$, number of scan: 32, range: 600$3800 \mathrm{~cm}^{-1}$ and mode: transmission,

2) The shapes and morphologies of the preparations were examined by transmission electron microscopy using JEOL, JEM-1230 transmission electron microscope, Tokyo, Japan. Drops of the diluted preparations were deposited on a carbon-coated copper grid, then left to dry at room temperature for $10 \mathrm{~min}$ before investigation.

3) The particle size distribution and zeta potential analysis were recorded at: run time: 2 min, temperature: $23^{\circ} \mathrm{C}$, solvent: water, concentration: $1 \mathrm{mg} / \mathrm{mL}$, using Gaussian/ Nicomp distribution analysis, particle sizing systems Inc. Santa Barbara, California, USA.

4) The quantitative analysis of the metals was determined using XRF Instrument: Axios, Sequential WD_XRF Spectrometer, PANalytical 2005.

5) Thermogravimetric analysis (TGA) of the prepared samples was carried out on PerkinElmer thermogravimetric analyzer TGA 7. The dry samples were heated from room temperature to $700^{\circ} \mathrm{C}$ at $5^{\circ} \mathrm{C} / \mathrm{min}$.

\section{$\underline{\text { Results and Discussion }}$}

Characterization of the crosslinked hydrogels

Figure 1 shows FTIR spectra of the prepared materials relative to chitosan and HBPE, separately. It was observed that the broad band at $3200-3500 \mathrm{~cm}^{-1}$ is attributed to $\mathrm{OH}$ stretching vibrations. In case of sample 2, HBPE crosslinked chitosan exhibited strong peaks at 2900 and 2850 $\mathrm{cm}^{-1}$ corresponding to $\mathrm{CH}_{3}$ and $\mathrm{CH}_{2}$ of HBPE. While, these peaks were decreased in case of samples 4, 5 and 6 due to the presence of metal complexes. The bands at 1652 and $1556 \mathrm{~cm}^{-1}$ are assigned to the carbonyl stretching of amide I bond and the $\mathrm{N}-\mathrm{H}$ bending vibrations of amide II bond, respectively [18].The results indicated that crosslinking of chitosan by HBPE was occurred.

Figure 2 shows TGA diagrams of the prepared crosslinked chitosan with HBPE relative to chitosan and HBPE, respectively. It was observed that the chitosan and HBPE are found in the thermograms of the prepared hydrogels with ratios $1: 1$ and 1:2 (samples 2 and 3). It is worthy to mention that the weight loss at temperature range less than $200^{\circ} \mathrm{C}$ is accomplished to the physically adsorbed water molecules. Moreover, in the case sample 2, at temperature region above $300^{\circ} \mathrm{C}$, the weight loss percentage of chitosan at $200-300^{\circ} \mathrm{C}(33 \%)$ is about nearly the same of $\mathrm{HBPE}$ at temperature region $300-400^{\circ} \mathrm{C}(33.6 \%)$. While in the case of sample 3 , the weight loss percentage of chitosan at $200-300^{\circ} \mathrm{C}$ $(24 \%)$ is about two times higher than that of HBPE at temperature region $300-400^{\circ} \mathrm{C}(40 \%)$. This is proved that the obtaining HBPE crosslinking with different ratios typically as was mentioned in the experimental section. On the other hand, the presence of metals $\mathrm{Co}$ or $\mathrm{Ni}$ exhibited catalytic degradation of the prepared hydrogels [19].

Figure $3 \mathrm{~A}$ and B show SEM-micrographs and TEM images of the prepared hydrogels, respectively. When the metals (Co, Ni and Fe) were reacted with the composites, the surfaces exhibited high porosity and the particles became more stable and well dispersed in the matrices. In case of sample 1, the morphology showed rough surface. While in case of sample 2 (1:1), HBPE crosslinked chitosan exhibited smoother surface. When the concentration of HBPE was increased, small particulates were obtained and covered the surface. From TEM images, chitosan particles exhibited heterogeneous colloid shapes due to HBPE crosslinking reaction. While, in case of presence of metals, they adsorbed in between the 


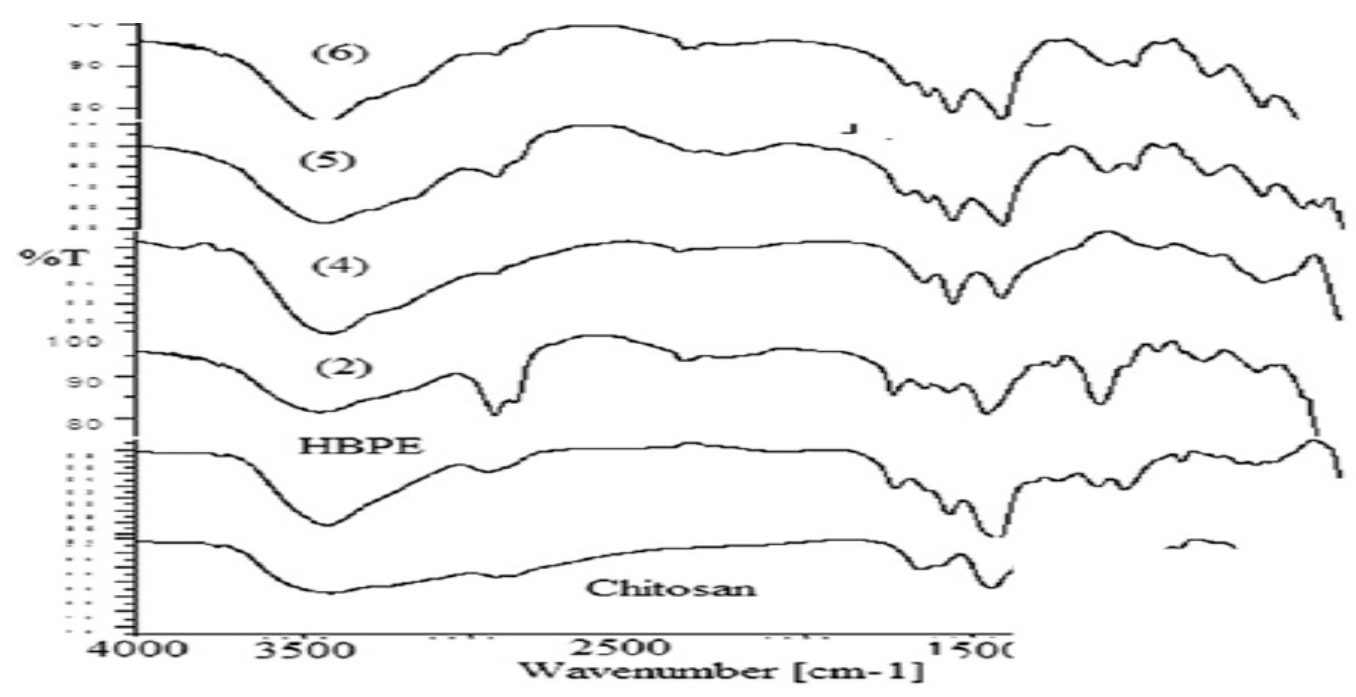

Fig 1. FTIR spectra of the prepared materials in comparison with chitosan and HBPE.

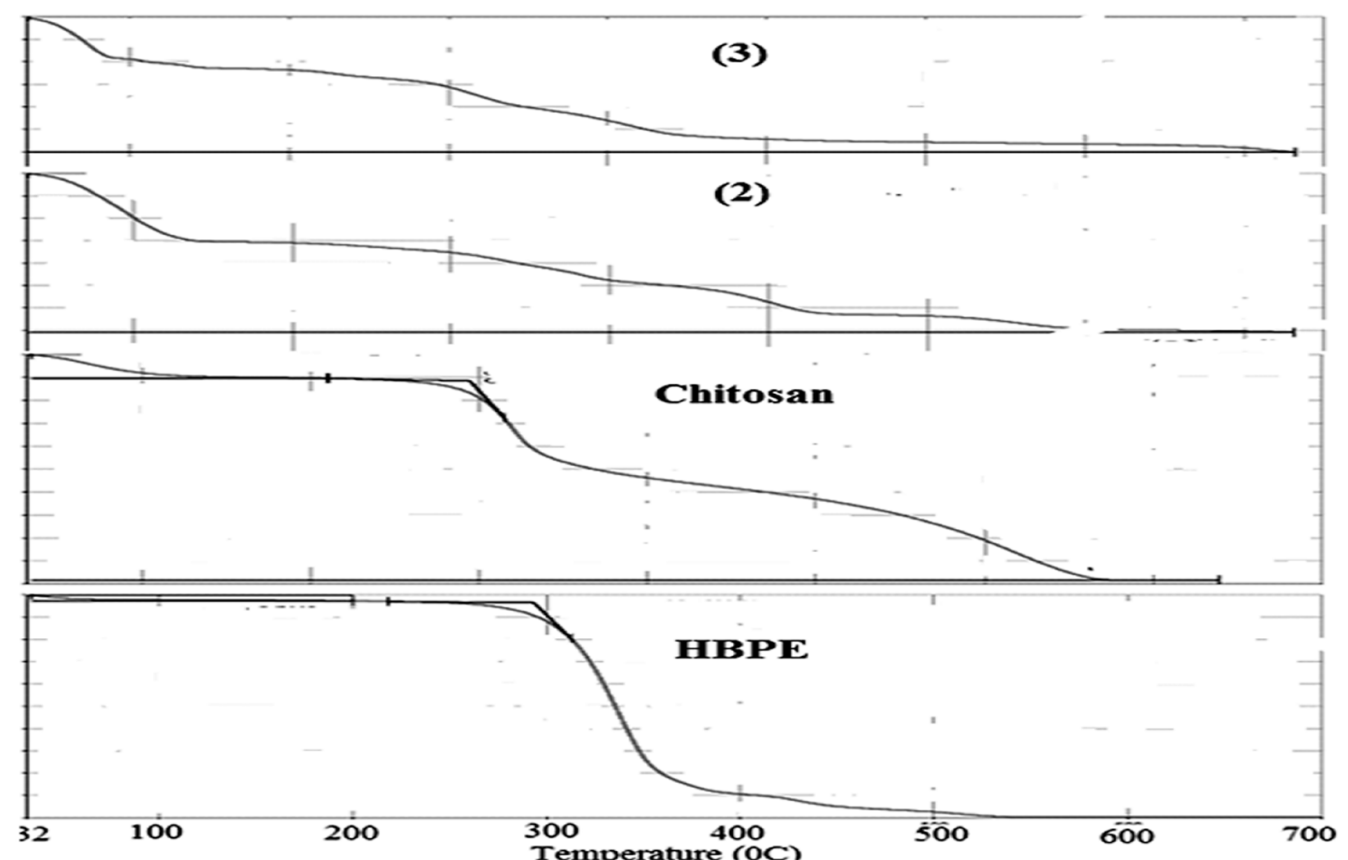

Fig 2. TGA diagrams of the prepared crosslinked chitosan with HBPE (2 and 3) relative to chitosan and $\mathrm{HBPE}$, respectively. 
(1)
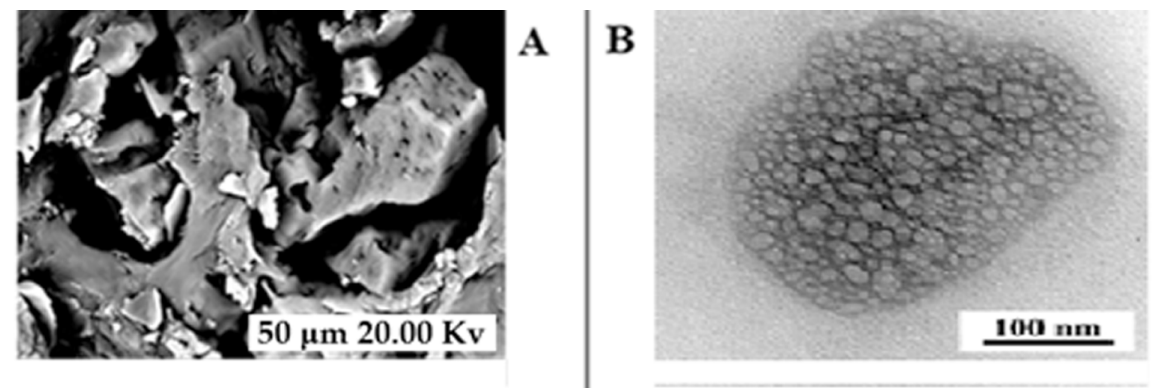

(2)

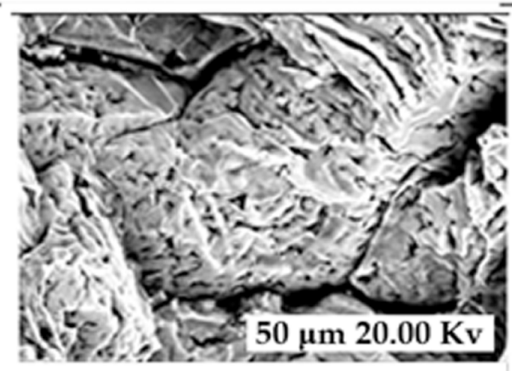

(3)

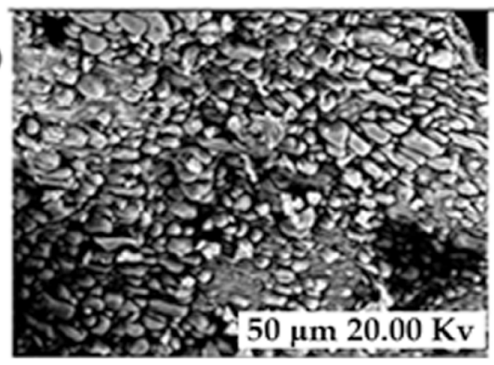

(4)

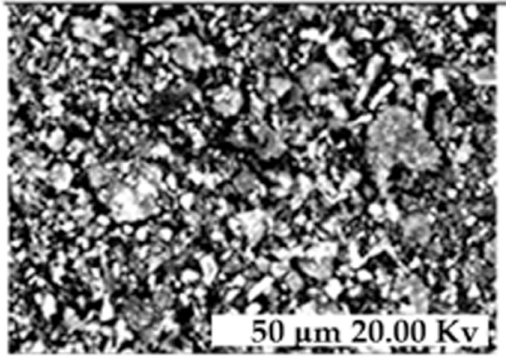

(5)
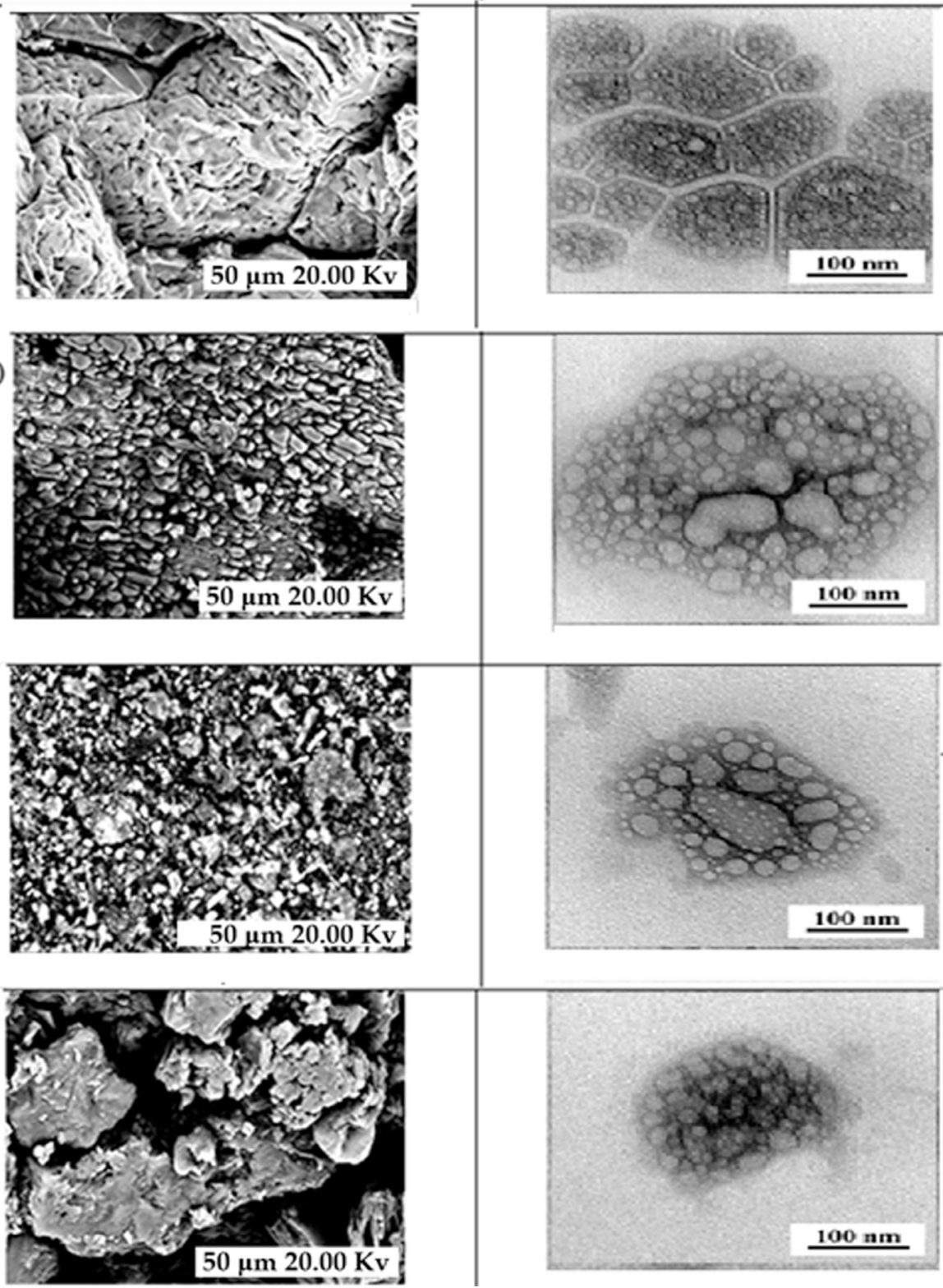

年 $20.00 \mathrm{Kv}$

Fig 3. (A) SEM-micrographs and (B) TEM images of the prepared materials. 
TABLE 1. Chemical composition of the prepared hydrogels

\begin{tabular}{|lccccc|}
\hline \multirow{2}{*}{ Sample Code } & \multicolumn{5}{c|}{ Chemical Composition (wt\%) } \\
\cline { 2 - 6 } & Chitosan & HBPE & Co & Ni & Fe \\
\hline$(1)$ & 20 & 10 & ----- & ----- & ------- \\
$(2)$ & 10 & 10 & ------ & ----- & ------ \\
$(3)$ & 10 & 20 & --- & ---- & ------ \\
$(4)$ & 10 & 10 & 10 & ---- & ----- \\
$(5)$ & 10 & 10 & ----- & 10 & 10 \\
$(6)$ & 10 & 10 & ---- & ----- & \\
\hline
\end{tabular}

TABLE 2. TGA data of the prepared hydrogels (2) chitosan/HBPE (1:1) and (3) chitosan/HBPE (1:2) relative to chitosan and HBPE separately.

\begin{tabular}{|c|c|c|c|c|}
\hline \multirow{2}{*}{ Sample Code } & \multicolumn{4}{|c|}{ Weight loss (wt\%) at different temperature range } \\
\hline & $35-200^{\circ} \mathrm{C}$ & $200-300^{\circ} \mathrm{C}$ & $300-400^{\circ} \mathrm{C}$ & $400-700^{\circ} \mathrm{C}$ \\
\hline Chitosan & \multicolumn{2}{|c|}{12.2} & \multicolumn{2}{|c|}{58.0} \\
\hline HBPE & \multicolumn{2}{|c|}{--------- } & \multicolumn{2}{|c|}{99.6} \\
\hline (2) & 33.0 & 33.0 & 33.6 & --------- \\
\hline (3) & 36.2 & 24.0 & 40.0 & -------- \\
\hline
\end{tabular}

individual stable colloidal shapes. It can be concluded that, the cobalt, nickel and iron metals were adsorbed at the surface and in between the network chains of the prepared hydrogels.

Figure 4 shows particle size and zeta potential analysis of the prepared samples 1,2 and 7 using DLS technique. The particle size of the prepared hydrogels with ratios $2: 1$ and 1:1 are around 139 and $118 \mathrm{~nm}$, respectively. While, the iron-complex has particle size around $136 \mathrm{~nm}$. It is clear that the particle size distribution analysis exhibited narrow peaks one modal in case of chitosan/HBPE and iron metal complex $(1: 1)$. However, in case of 2:1 ratio, a bimodal of particle size distribution had been observed. This may be attributed to the increasing content of chitosan, which has an anionic $-\mathrm{OH}$ solubilizing groups with high PDI value $(0.5)$ in comparison with that in case of the similar ratio $(0.271)$. On the other hand, all zeta potential had negative values, which indicates that the prepared hydrogels were obtained with good stability particles. In other words, iron-complex hydrogel had high negative zeta potential value $(-49 \mathrm{mv})$. It is assumed that the high zeta potential particles is more electrically stable than those lower values which tend to obtain flocculating and agglomerating particles with time. This assumption is arisen from the charged shell around the hydrogel particles, which is prohibited the particle-particle interaction and increased the dispersion stability. This was confirmed in terms of PDI values which are little changed in between 0.192 and 0.536 in case of iron-complex and uncomplexed hydrogel.

\section{Metal-binding behaviour of the prepared hydrogels}

According to the previous work [20], in case of using HBPE with low generation $(16-\mathrm{OH})$ as crosslinker agent, the metal ions could be easily diffused into the interior of the HBPE and to be adsorbed during the complex reaction. In spite of chitosan was not a suitable adsorbent because of the fact it was soluble in aqueous acidic solution and could not be recovered, crosslinked chitosan with HBPE could be used in acidic solution as well as its adsorption capability was improved.

Figure 5 and Table 3 show XRF data analysis of the prepared $\mathrm{Co}, \mathrm{Ni}$ and $\mathrm{Fe}$ metal complex hydrogels. They demonstrated that the metal complex of the crosslinked chitosan with HBPE had been successful prepared. In other words, Co, 
TABLE 3. XRF Analysis Results of the prepared metal complex hydrogels

\begin{tabular}{|lccc|}
\hline Main Constituents in (wt\%) & $\mathbf{( 4 )}$ & $\mathbf{( 5 )}$ & (6) \\
\hline $\mathrm{Fe}$ & 0.06 & 0.04 & 6.56 \\
$\mathrm{Ni}$ & 0.02 & 7.01 & 0.01 \\
$\mathrm{Ca}$ & 0.06 & 0.11 & 0.11 \\
$\mathrm{Na}$ & 15.10 & 19.85 & 17.67 \\
$\mathrm{~K}$ & 0.02 & 0.02 & 0.01 \\
$\mathrm{Co}$ & 6.55 & 0.01 & -- \\
$\mathrm{LOI}$ & 77.52 & 72.34 & 74.70 \\
\hline
\end{tabular}

TABLE 4. Particle size and zeta potential measurements using DLS technique.

\begin{tabular}{|lccc|}
\hline Sample code & Particle size (nm) & Zeta potential (mv) & PDI (Variance) \\
\hline$(1)$ & $118 \pm 70$ & -6.88 & 0.536 \\
$(2)$ & $139 \pm 67.6$ & -11.8 & 0.271 \\
$(3)$ & $582 \pm 430$ & 17.17 & 0.554 \\
$(4)$ & $679 \pm 495$ & 29.71 & 0.531 \\
$(5)$ & $509 \pm 330$ & -36.1 & 0.419 \\
$(6)$ & $136 \pm 54.2$ & -49.3 & 0.192 \\
\hline
\end{tabular}

hydrogel particles and the composites showed more

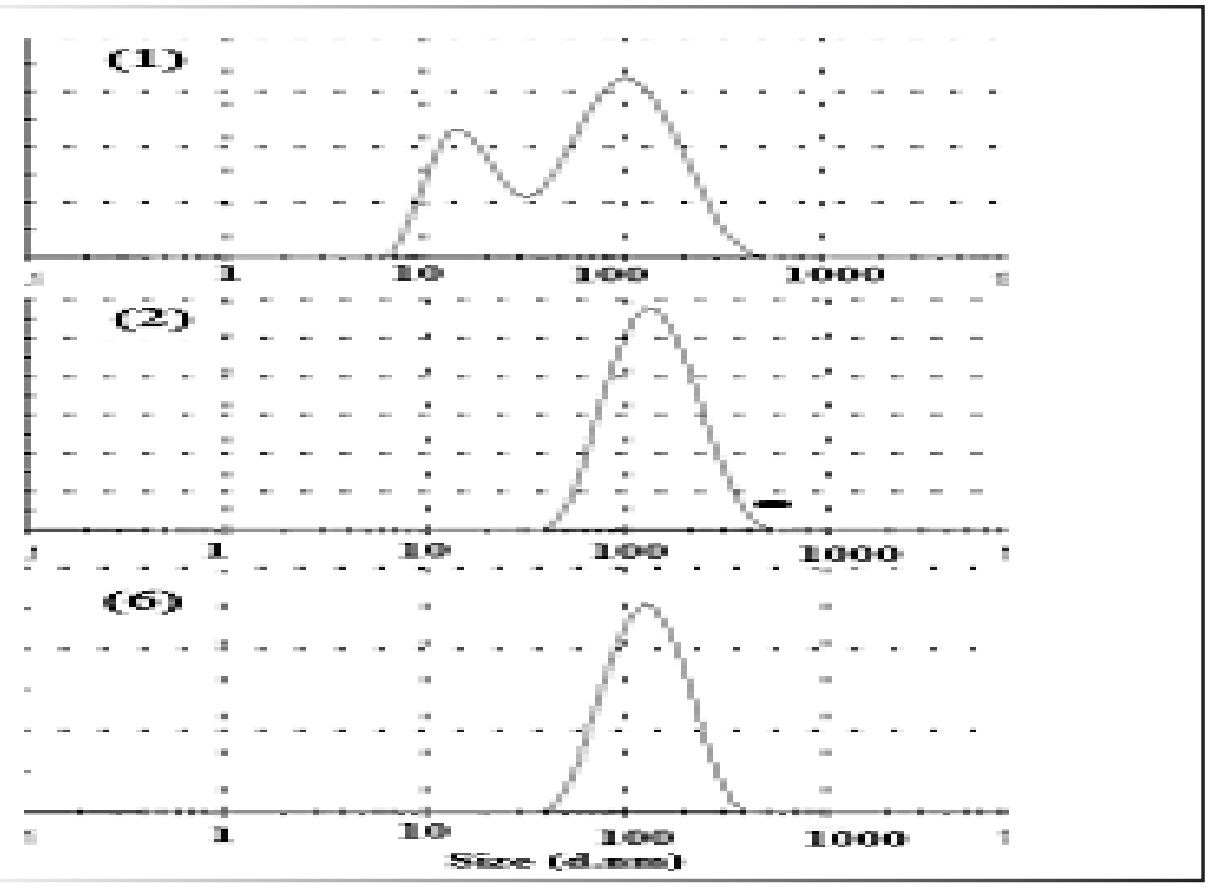

Fig 4. Particle size distribution and zeta potential analysis of the prepared materials using DLS technique. 

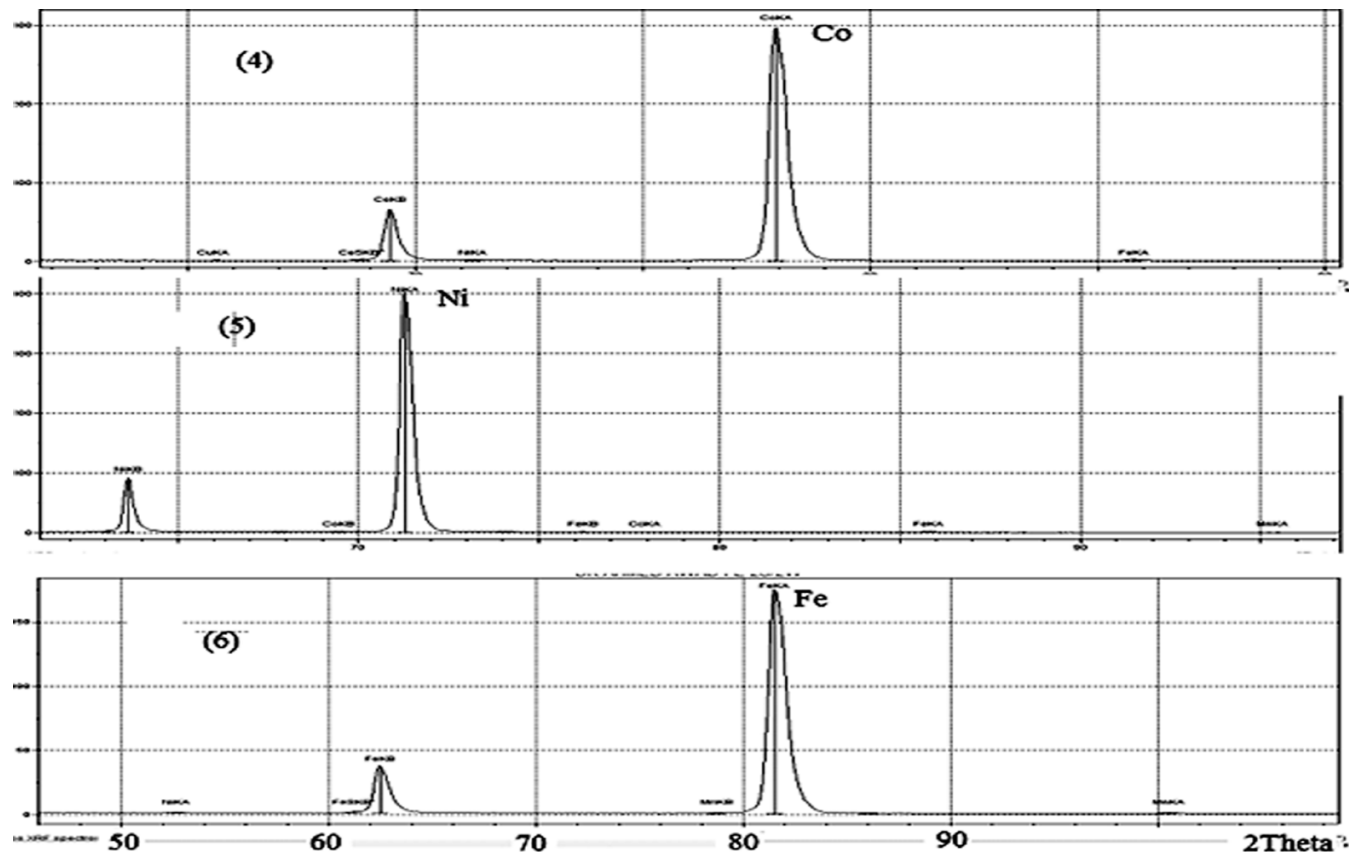

Fig 5 . XRF diagrams of the prepared metals complex hydrogels.

$\mathrm{Ni}$ and Fe percentages were decreased to 6.55 , 7.01 and $6.56(\mathrm{wt} \%)$ in the prepared samples relative to the initial metal concentrations (10 wt $\%$, respectively.

\section{Conclusions}

Metal complex chitosan hydrogels crosslinked with HBPE generation 2, were successfully prepared in different ratios. The characterization gave supporting evidence for crosslinking of chitosan with typical ratios $(1: 1$ and $1: 2)$ as was mentioned in the experimental part. Moreover, the different metals $\mathrm{Co}, \mathrm{Ni}$ and $\mathrm{Fe}$ were complexed with the hydrogels. The morphological observations indicated the presence of embedded metal ions. The prepared materials could be used as colour adsorbents. In other words, these complexed hydrogels have multifunctional application modes after further investigations.

\section{Acknowledgment}

Authors wish to thank The Egyptian Academy of Scientific Research \& Technology, Cairo, Egypt (ASRT), Czech Academy of Sciences, Prague, Czech Republic (CAS) and Grant Agency of the Czech Republic (project 17-04258J) for financial supporting of this work according to the Bilateral
Joint Project Funding Call 2016/2018.

\section{References}

1.Liu P., Hyperbranched polymers grafted inorganic nanoparticles. A leterature Review. Mater Res Innov. 9,103 (2005).

2. Liu P., Tian J., Liu MW., Xue QJ. Fabrication dendrimer-like PAMAM based on silica nanoparticles..Chin. J. Chem 21, 960 (2003).

3. Bosman A.W., Janssen H.M., Meijer E.W. Chem Rev 99, 1665 (1999).

4. Thomasson D., Boisson F., Girard-Reydel E., Mechin F. Hydroxylated hyperbranched polyesters as crosslinking agents for polyurethane networks: Partial modification of the $\mathrm{OH}$ chain ends.Reactive and Functional Polym 66, 1462 (2006).

5. Varma, A.J., Deshpande, S.V., Kennedy JF. Carbohydr Polym 55, 77 (2004).

6. Cestari AR, Vieira EF, Santos AG,.Mota JA, Almeida VP. J. Colloid Interface Sci. 280, 380 (2004).

7. Elhalawany, N., Haroun A., Abbas, H, Hammad F.Conducting chelating polymer composites based on grafted waste polystyrene for removal of toxic copper ions. J. Elastomers and Plastics 46, 553 (2014).

Egypt.J.Chem. 60 , No. 5 (2017) 
8.Haroun A.A., Mashaly HM, El-Sayed NH. Novel nanocomposites based on gelatin/HPET/chitosan with high performance acid red 150 dye adsorption. Clean Technologies and Environmental Policy15, 367 (2013).

9. Haroun A.A., Mashaly H.M., Helmy H.M,.Kamel M.M. Kinetic study of gelatin/chitosan based nanocomposites for acid red 150 dye adsorption using ultrasonic energy. Egypt. J. Chem. 60, 41 (2017).

10. Youssef A.M., Abou El FettouhA.Abd ElHakim, Malhat M.F, Haroun A.A. Free emulsion polymerization of styrene/layered double hydroxide nanocomposite for $\mathrm{Cd}$ and $\mathrm{Pb}$ (II) ions removal from aqueous media.Nano Science and Nano Technology: An Indian J7, 116. (2013).

11. Haroun A.A, El-Halawany NR. Preparation and evaluation of novel interpenetrating polymer network-based on newspaper pulp for removal of copper ions.Polym-Plastics Technol and Eng 50, 232 (2011).

12. Haroun A.A.,Abu Taleb EM, Abd El-Ghaffar MA. Synthesis and characterization of novel thermoplastic films for removal of heavy metal ions. Polym-Plastics Technol and Eng 49,454 (2010).

13.Wan Ngah WS.,Endud CS., Mayanar R. React. Funct.Polym 50, 181 (2002).
14.Mi FL, Chyn SS., Chen CT, Lai JY. Polymer 43, 757 (2002).

15.Cobianco S.,Lezzi A., Scotti R.A. Spectroscopic study of $\mathrm{Cu}$ (II)-complexes of chelating resins containing nitrogen and sulfur atoms in the chelating groups. React. Funct. Polym. 43, 7 (2000).

16.Gonzalez M.E.L.,Arribas LVP. Chemically modified polymeric sorbents for sample preconcentration. $J$ ChromatogrA 902, 3 (2000).

17. Antonietti M, Landfester K. Polyreactions in miniemulsions. Proc. Polym. Sci 27, 689 (2002).

18. Tien C.L, Lacroix M., Ispas-Szabo P., Mateescn MA.N-acetylated chitosan: hydrophobic materials for controlled drug release. J. Controlled Release 93, 1 (2003).

19. Ou C., Li S, Shao J., Fu T., Liu Y.,Fan W., Yang X., Bi X. Cogent Chemistry 2,1216247 (2016).

20. Qu R., Sun C.,.Ji C, Wang C., Chen H., Niu Y., Liang C., Song Q. Preparation and metal-binding behaviour of chitosan functionalized by ester-and amino-terminated hyperbranched polyamidoamine polymers.Carbohydrate Res. 343, 267 (2008).
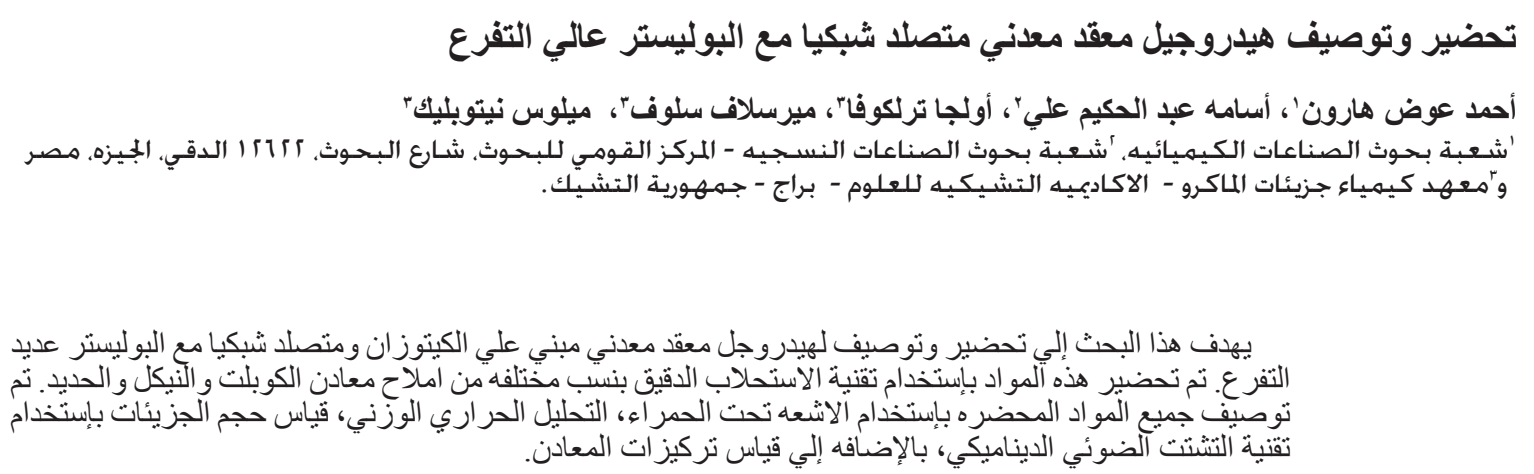